\title{
Uber die Auslösung von Ultrastrahlungsschauern in Blei
}

\author{
Von H. Faissner, K. H. Lauterjung und B. Schimmer \\ Aus dem Institut für Physik im Max-Planck-Institut für medizinische Forschung, Heidelberg \\ (Z. Naturforschg. 11 a, 549-555 [1956]; eingegangen am 20. April 1956)
}

\begin{abstract}
Die Auslösung von Ultrastrahlungsschauern in Blei wurde im Bereich von 0 bis $36 \mathrm{~cm} \mathrm{~Pb}$ untersucht. Die Schauer wurden $1 \mathrm{~m}$ unterhalb des Schauerstrahlers mit zwei gekreuzten Zählrohrlagen nach Thurs ${ }^{2}$ oder mit einer Lage paralleler Zählrohre nachgewiesen. Eine oder zwei zusätzliche Zählrohrlagen waren unterhalb und oberhalb bzw. an verschiedenen Stellen innerhalb des Schauerstrahlers angebracht. Durch geeignete Koinzidenz-Antikoinzidenz-Kombinationen konnten bestimmte Schauertypen gesondert untersucht werden. Die Häufigkeit der bei Bleidicken über $10 \mathrm{~cm}$ erzeugten Schauer nahm mit zunehmender Bleidicke langsam und monoton ab. Ihre scheinbare Absorptionslänge ergab sich zu $(210 \pm 30) \mathrm{cm} \mathrm{Pb}$. Sie wurden ausschließlich durch ionisierende Teilchen ausgelöst. Die abgeschätzte Absoluthäufigkeit wäre mit der Annahme verträglich, daß es sich hierbei im wesentlichen um $\mu$-Mesonen mit begleitenden Elektronen handelt. Höhere Maxima zeigten sich nicht.
\end{abstract}

\section{Problemstellung und allgemeine Anordnung}

In einer vorangegangenen Arbeit ${ }^{1}$ wurde gezeigt, daß bei einer Zählrohranordnung nach THURN ${ }^{2-4}$ keine außerhalb der Fehler liegenden höheren Maxima der Rossi-Kurve auftreten. Dabei befanden sich zwei gekreuzte Lagen von je 12 Zählrohren ungefähr $1 \mathrm{~m}$ unterhalb des Schauerstrahlers aus Blei; die Divergenzwinkel lagen um $3,4^{\circ}$. Wegen des hohen Untergrundes von Seitenschauern und schrägen Einzelstrahlen konnte für die Überhöhung der fraglichen Maxima nur eine obere Schranke von $20 \mathrm{Ko} / \mathrm{h}$ gesetzt werden.

Durch die in der vorliegenden Arbeit beschriebenen Versuche sollte nachgeprüft werden, ob höhere Maxima mit einer damit verträglichen UUberhöhung existieren. Dazu wurden zusätzliche Zählrohrlagen unterhalb und oberhalb (Ziff. 2) bzw. innerhalb (Ziff. 3) des Schauerstrahlers angebracht und mit den gekreuzten Lagen in Koinzidenz geschaltet. Dadurch wurde der Untergrund größtenteils ausgeschaltet. Außerdem ließen sich mit geeigneten Koinzidenz - Antikoinzidenz-Kombinationen Vorstellungen über die stufenweise Ausbildung der fraglichen Schauer ${ }^{2,5}$ genauer kontrollieren. Das Ergebnis der unter Ziff. 3 beschriebenen Versuche wurde bereits kurz mitgeteilt ${ }^{6}$.

1 H. Faissner, K. H. Lauteruung u. B. Schimmer, Z. Naturforschg. 11 a, 315 [1956].

2 H. Thurn u. W. Bothe, Z. Naturforschg. 6 a, 576 [1951].

3 H. Thurn, Z. Naturforschg. 7 a, 497 [1952].

${ }^{4}$ H. Thurn, Z. Naturforschg. 8 a, 134 [1953].

5 W. Bothe u. H. Krämer, Phys. Rev. 94, 1402 [1954].

${ }^{6}$ H. Faissner u. W. Bothe, Z. Naturforschg. 10 a, 794 [1955].

7 M. Della Corte, Atti Accad. Naz. Lincei (R. C. Cl. Sci. Fis. Math. Nat.) (8) 1, 974 [1946].
Die Registrierung der Vierfachkoinzidenzen aus den gekreuzten Lagen wurde früher näher beschrieben ${ }^{1}$. Die Koinzidenzen bzw. Antikoinzidenzen der Vierfachkoinzidenzen mit den Zusatzlagen wurden durch Zweifach-Rossi-Stufen mit nachfolgendem Sснмітт-Trigger ausgesondert. Außerdem waren die Zusatzlagen direkt mit den Zweifachkoinzidenzen aus jeder der gekreuzten Lagen in Koinzidenz geschaltet. Die Schauerwinkel waren dann nach oben nur durch die Zählrohrlänge begrenzt und Schauerhäufigkeit und statistische Genauigkeit der Messung entsprechend größer. Eine Anordnung mit parallelen Zählrohren und einer Zusatzlage dicht unter dem Schauerstrahler wurde auch von anderen Autoren benutzt ${ }^{7-12}$. In Ziff. 4 wurde eine Anordnung nach Noor ${ }^{13}$ verwendet, bei der ebenfalls dicht unterhalb des Schauerstrahlers ionisierende Schauerteilchen verlangt worden waren.

Die Koinzidenzauflösezeit betrug in Ziff. $25 \mu \mathrm{sec}$, sonst $20 \mu$ sec. Die Zufallskoinzidenzen waren daher bei den meisten Messungen zu vernachlässigen. Der Einfluß des Luftdrucks war klein. Er mittelte sich heraus, da die Dicke des Schauerstrahlers im Vergleich zu Luftdruckänderungen sehr rasch variiert wurde.

8 J. Clay, A. Venema u. K. H. Jonker, Physica 7, 673 [1940].

9 J. Clay U. W. L. Scheen, Physica 14, 489 [1948].

10 J. Clay, Rev. Mod. Phys. 21, 82 [1949].

11 J. Clay u. G. Klein, Physica 16, 293 [1950].

12 L. Allegretti u. Abd El-W ahab Khalil, Nuovo Cim. 8, 1011 [1951].

13 M. G. Noон u. H. Авu Zeid, private Mitteilung. Wir danken Herrn Prof. Nоон für die vorzeitige Mitteilung seiner Resultate. 


\section{Zusätzliche Zählrohrlagen unterhalb und oberhalb des Schauerstrahlers}

Die Apparatur befand sich bei diesen Messungen im Dachgeschoß des Instituts unter einer Luke, die entweder offen oder mit $0,5 \mathrm{~g} / \mathrm{cm}^{2}$ Aluminium bedeckt war. In den gekreuzten Lagen I und II wurden jeweils Zweifachkoinzidenzen zwischen allen benachbarten Zählrohren registriert. Bei den ersten Messungen war eine Zusatzlage III von 24 parallel geschalteten Zählrohren von $2 \mathrm{~cm}$ Durchmesser $5 \mathrm{~cm}$ unterhalb des Schauerstrahlers angebracht. Die effektive Zählrohrlänge betrug bei allen Messungen in Ziff. 2 und 3 rd. $60 \mathrm{~cm}$. Wie in Abb. 1 skizziert, wurden alle Schauer registriert, die von geladenen oder neutralen Primären im Schauerstrahler ausgelöst wurden. Seitenschauer waren bei Bleidicken über $5 \mathrm{~cm}$ weitgehend ausgeschaltet.

Die registrierten Fünffachkoinzidenzen (I, I, II, II, III) zeigt Abb. 1 a. Die Kurve hat ein scharfes Rossı-Maximum bei $1 \mathrm{~cm} \mathrm{~Pb}$ und fällt bei größeren Bleidicken monoton ab. Der Nullpunkt ist durch Deckenschauer hochgedrückt. Die Überhöhung des Rossi-Maximums über dem praktisch geradlinigen Teil der Kurve ist $(250 \pm 10) \mathrm{Ko} / \mathrm{h}$, also genau so groß wie in der Kurve der Vierfachkoinzidenzen
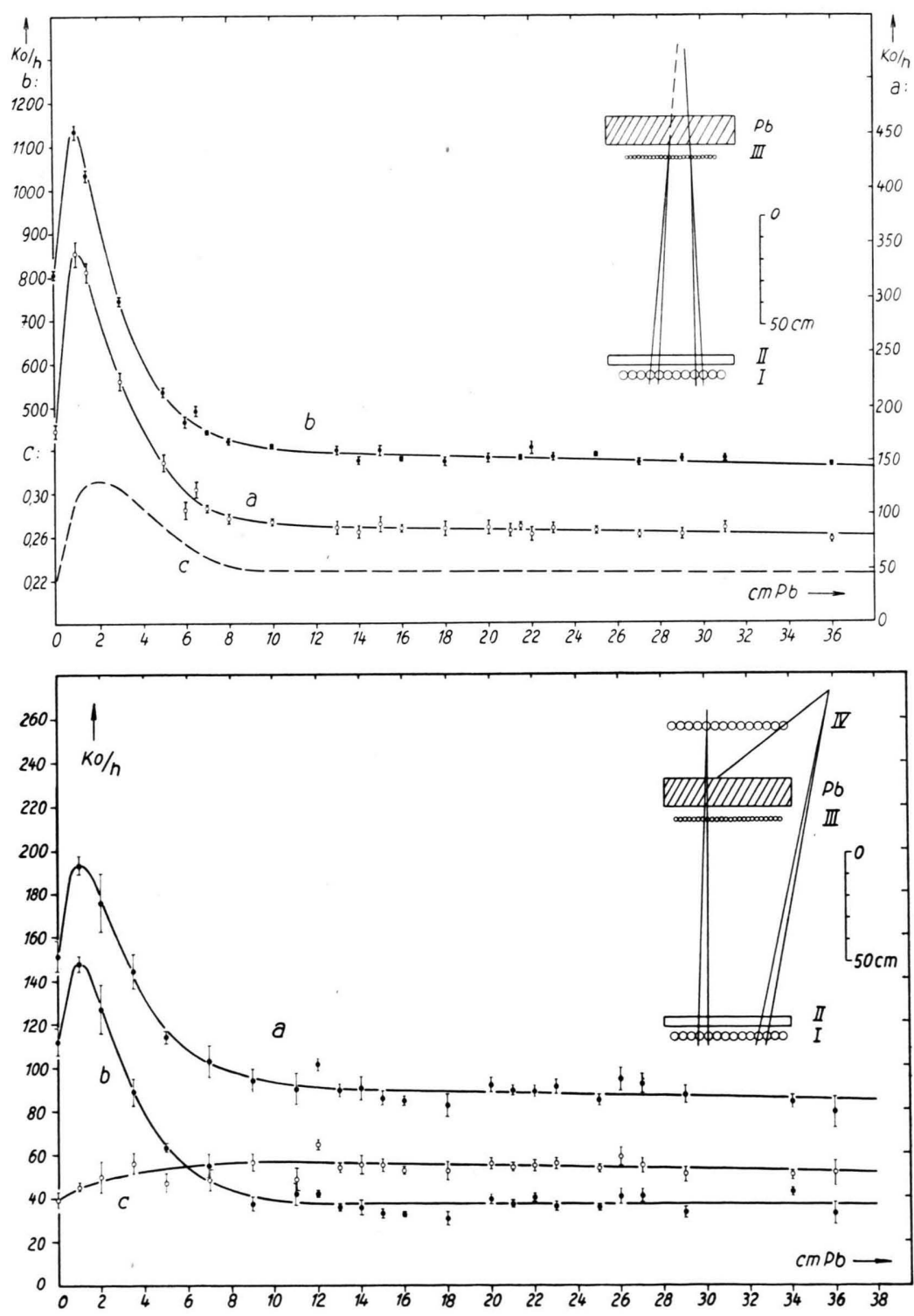

Abb. 1. Rossi-Kurven für Schauer, die $5 \mathrm{~cm}$ unterhalb des Schauerstrahlers ionisieren. a) Koinzidenzen (I, I, II, II, III). b) Mittel aus (I, I, III) und (II, II, III). c) Quotient : Kurve a) durch Kurve b).
Abb. 2. Rossi-Kurven mit gekreuzten Zählrohrlagen. a) Mindestens 1 geladenes Teilchen oberhalb des Schauerstrahlers: (I, I, II, II, IV). b) Durchgehend ionisierende Schauer: (I, I, II, II, III, IV). c) Seitenschauer: (I, I, II, II, - III, IV). 
(I, I, II, II) (l. c. ${ }^{1}$, Abb. 2 a). Die absolute Koinzidenzrate ist dagegen stark reduziert: Sie beträgt im Rossi-Maximum 9\% und oberhalb $10 \mathrm{~cm} \mathrm{~Pb}$ nur $2,8 \%$ der Vierfachkoinzidenzrate. Für isotrop einfallende Strahlung müßte sie etwa $8 \%$ sein. Nur im Rossi-Maximum ist also die Vertikale bevorzugt: bei größeren Bleidicken wurde der weitaus größte Teil der Vierfachkoinzidenzen durch schräge Einzelstrahlen und Seitenschauer verursacht (vgl. die Abschätzung in Anm. ${ }^{1}$, Ziff. 3).

Die Dreifachkoinzidenzraten (I, I, III) und (II, II, III) stimmten innerhalb der Fehler überein. Sie wurden deshalb gemittelt (Abb. 1 b). Bei der Berechnung des eingezeichneten mittleren statistischen Fehlers wurde berücksichtigt, daß die beiden Koinzidenzraten nicht unabhängig sind. Das RossiMaximum liegt auch hier bei $1 \mathrm{~cm} \mathrm{~Pb}$.

Der Quotient: Kurve 1 a durch Kurve 1 b (Abb. 1 c) gibt die Wahrscheinlichkeit an, mit der ein als Dreifachkoinzidenz registrierter Schauer auch als Fünffachkoinzidenz gezählt wird. Diese Wahrscheinlichkeit hängt von den Divergenzwinkeln und der Multiplizität der Schauer ab. Für sehr teilchenreiche Schauer ist sie praktisch gleich 1 . Kurve $1 \mathrm{c}$ hat ein breites Maximum bei $2 \mathrm{~cm} \mathrm{~Pb}$. Die ElektronenPhotonen-Kaskaden haben sicher keine kleineren Divergenzwinkel als die bei größeren Bleidicken ausgelösten Schauer. Durch dieses Maximum wird also ihre größere Multiplizität angezeigt.

Nach den Messungen von ThurN ${ }^{2,4}$ sollten die Schauer des 2. Maximums durch Ionisierende ausgelöst werden und nach denen von $\mathrm{KRÄMER}^{5}$ dicht unterhalb des Schauerstrahlers neutral sein. Man sollte also das isolierte 2. Maximum erhalten, wenn man eine Zählrohrlage III dicht unterhalb des Bleis in Antikoinzidenz und eine weitere Lage IV oberhalb des Bleis in Koinzidenz mit den in den gekreuzten Lagen I/II nachgewiesenen Schauern schaltet. Das wurde mit der in Abb. 2 skizzierten Anordnung geprüft. Die Lage IV bestand aus 14 parallel geschalteten Zählrohren von $4 \mathrm{~cm}$ Durchmesser, und war in einem konstanten Abstand von $37 \mathrm{~cm}$ oberhalb der Bleiunterseite angebracht. Der Raumwinkel für die ionisierenden Primären war also für alle Meßpunkte konstant.

Simultan wurden registriert: Fünffachkoinzidenzen (I, I, II, II, IV) (Kurve $2 \mathrm{a}$ ), deren Koinzidenzen mit III: (I, I, II, II, III, IV) (Kurve 2 b) und deren Antikoinzidenzen mit. III: (I, I, II, II, - III, IV) (Kurve $2 \mathrm{c}$ ). Da es sich um eine Alternative handelt, muß die Summe von Sechsfachkoinzidenzen $2 \mathrm{~b}$ und Antikoinzidenzen $2 \mathrm{c}$ exakt die Fünffachkoinzidenzen 2 a ergeben. Das ist für jeden $\mathrm{Me}$ punkt in guter Näherung der Fall.

Kurve 2 b zeigt, wie zu erwarten war, das RossiMaximum über einem kleinen, annähernd konstanten Untergrund. Ein gleich hohes Rossi-Maximum über einem stärkeren Untergrund weist Kurve 2 a auf. Weder in Kurve 2 a noch in Kurve 2 c tritt ein 2. Maximum auf. In Kurve $2 \mathrm{c}$ wurden offenbar nur Seitenschauer erfaßt, bei denen ein Begleitstrahl zwar die Lage IV, nicht aber die Lage III traf (s. Skizze). Der leichte Anstieg auf den ersten cm $\mathrm{Pb}$ erklärt sich so zwanglos. Die ebenfalls mitgemessenen Koinzidenzen (II, II, IV) und (II, II, III, IV) zeigen denselben Verlauf wie die entsprechenden Kurven $2 \mathrm{a}$ und $2 \mathrm{~b}$.

\section{Messungen mit einer Zusatzlage innerhalb des Schauerstrahlers}

Bei diesen Messungen war die Apparatur im ersten Obergeschoß des Instituts unter ca. $70 \mathrm{~g} / \mathrm{cm}^{2}$ Betondecken aufgestellt. Die Zählrohre in den gekreuzten Lagen I und II waren wie bei Thurn $^{2}$ in Dreiergruppen zusammengefaßt (Standardanordnung ${ }^{1}$ ). Eine Zusatzlage III von 26 Zählrohren von $2 \mathrm{~cm}$ Durchmesser bzw. 15 Zählrohren von $4 \mathrm{~cm}$ Durchmesser war an verschiedenen Stellen innerhalb des Schauerstrahlers angebracht. Das geschah aus folgender Überlegung heraus: Die für die höheren Maxima verantwortlichen Schauer könnten von neutralen Primären erzeugt werden und neutral aus dem Blei austreten. Dann sollten wenigstens im Verlauf des Auslöseprozesses ionisierende Sekundärstrahlen auftreten.

Bei der ersten Meßreihe befand sich die Lage III konstant $5 \mathrm{~cm}$ oberhalb der Bleiunterseite. Bei den zur Kontrolle mitgemessenen Meßpunkten unterhalb $5 \mathrm{~cm}$ lag sie auf dem Blei auf. Der erfaßte Raumwinkel war also oberhalb $5 \mathrm{~cm} \mathrm{~Pb}$ exakt und unterhalb $5 \mathrm{~cm} \mathrm{~Pb}$ beinahe konstant. Die Kurve der Fünffachkoinzidenzen (I, I, II, II, III) (Abb. 3 a) zeigt das Rossi-Maximum und einen bei großen Bleidicken annähernd konstanten Untergrund. Der flachere Abfall des Rossi-Maximums ist durch die Absorption der Begleitstrahlen weicher Seitenschauer bedingt. Höhere Maxima treten auch hier nicht auf. Die Dreifachkoinzidenzen (II, II, III) (Kurve 3 b) zeigen den entsprechenden Verlauf.

Weitere Kurven wurden mit einer konstanten Blei- 


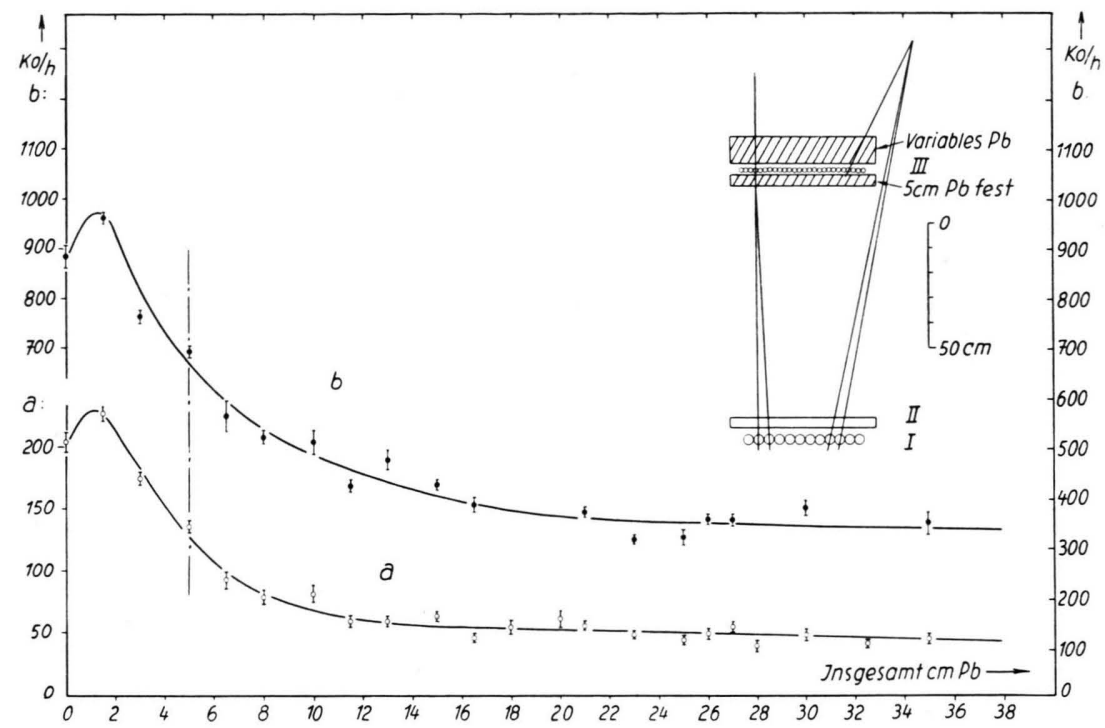

Abb. 3. Rossi-Kurven für Schauer, die $5 \mathrm{~cm}$ oberhalb der Bleiunterseite ionisieren. a) Koinzidenzen (I, I, II, II, III) . b) Koinzidenzen (II, II, III) .

schicht von $10 \mathrm{~cm}$ Dicke unter den Zählrohren III gemessen. Sie fallen bei Berücksichtigung des veränderten Raumwinkels mit den Kurven der Abb. 3 zusammen, wenn man als Abszisse in beiden Fällen die Bleidicke über der Lage III aufträgt. Der von den weichen Seitenschauern verursachte Abfall der Kurven erstreckt sich hier bis in den interessierenden Bereich um $15 \mathrm{~cm} \mathrm{~Pb}$. Deshalb wurde bei den folgenden Messungen die Bleischicht oberhalb der Zählrohrlage III konstant gehalten und die Schichtdicke darunter variiert. Die Zahl der mitgemessenen Seitenschauer ist nun von der Bleidicke unabhängig. Dagegen ändert sich der erfaßte Raumwinkel.

Die mit einer konstanten Bleischicht von $3 \mathrm{~cm}$ oberhalb der Zählrohre III gemessene Kurve der Fünffachkoinzidenzen (I, I, II, II, III) gibt Abb. 4 a wieder. Sie fällt oberhalb $3 \mathrm{~cm} \mathrm{~Pb}$ monoton ab. Bei der Korrektur auf konstanten Raumwinkel ist $\mathrm{zu}$ beachten, daß die Seitenschauer $(\approx 15 / \mathrm{h})$ und $\mathrm{Zu}$ fallskoinzidenzen $(\approx 3 / \mathrm{h})$ praktisch raumwinkelunabhängig sind. Die korrigierte Kurve $4 \mathrm{~b}$ verläuft bei großen Bleidicken wieder fast horizontal. Unterhalb $3 \mathrm{~cm} \mathrm{~Pb}$ werden zusätzliche Seitenschauer mitgemessen (schraffierte Fläche). In Abb. $4 \mathrm{c}$ ist das Mittel aus den Dreifachkoinzidenzraten (II, II, III) und (I, I, III) aufgetragen. Abb. $4 \mathrm{~d}$ zeigt die wie oben korrigierte Kurve. Höhere Maxima zeigen sich in keiner der Kurven.

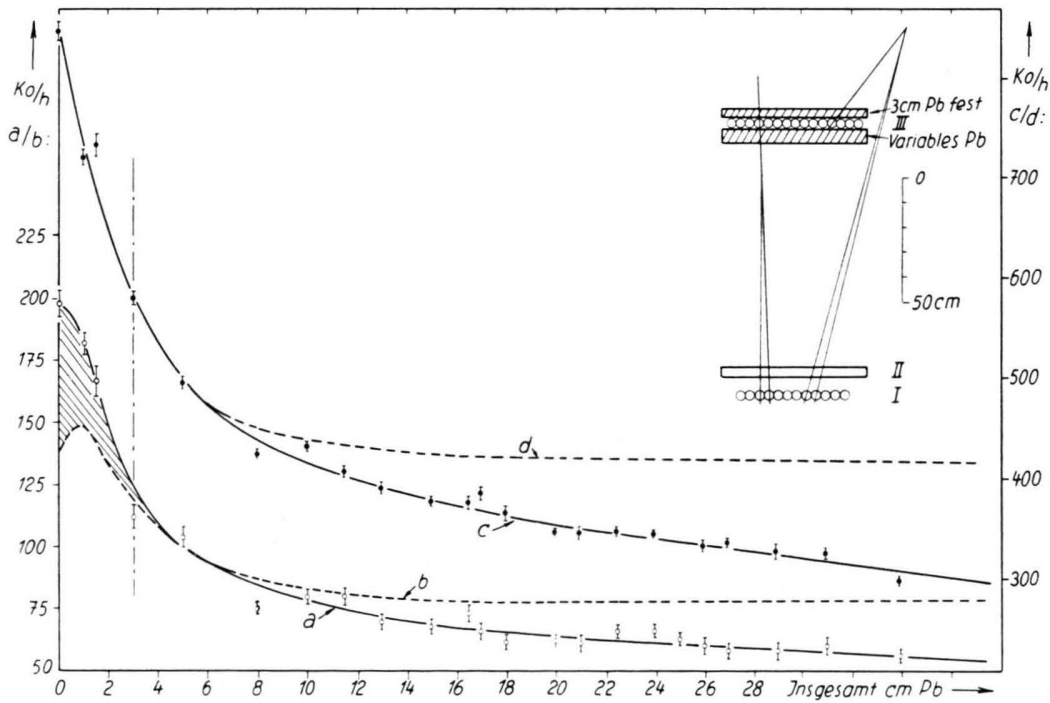

Abb. 4. Rossi-Kurven für Schauer, die $3 \mathrm{~cm}$ unterhalb der Bleioberfläche ionisieren. a) Koinzidenzen (I, I, II, II, III). b) Auf Raumwinkeländerung korrigierte Kurve a. c) Mittel aus den Koinzidenzen (II, II, III) und (I, I, III). d) Auf Raumwinkeländerung korrigierte Kurve c. 
Bei einer Meßreihe mit $1 \mathrm{~cm} \mathrm{~Pb}$ oberhalb der Zählrohrlage III ergaben sich, bis auf die erwartete Verschiebung des Untergrundes, die gleichen Kurven wie zuvor. In einer weiteren Meßreihe wurde die Bleischicht oberhalb III bei einer konstanten Gesamtbleidicke von $15 \mathrm{~cm}$ variiert. Die Koinzidenzraten zeigten mit zunehmender Bleidicke oberhalb III erst den von der Absorption der weichen Seitenschauer herrührenden Abfall und dann einen durch die Vergrößerung des Raumwinkels bedingten Wiederanstieg. Auch hier waren keine Anzeichen für die Auslösung von bevorzugt bei $15 \mathrm{~cm} \mathrm{~Pb}$ auftretenden Schauern zu finden.

\section{Anordnung mit bevorzugt vertikalem Strahlengang}

Die Anordnung ist in Abb. 5 skizziert. Lage II bestand aus 7 alternierend parallel geschalteten Zählrohren von $2,4 \mathrm{~cm}$ Durchmesser und $28 \mathrm{~cm}$ Länge. Die dicht unterhalb des Bleis angebrachten Zählrohre III hatten eine wirksame Zählfläche von $16 \times 40 \mathrm{~cm}^{2}$ und waren nach allen Seiten durch $10 \mathrm{~cm}$ dicke Bleiabsorber geschützt. Vier parallel geschaltete Zählrohre I mit einer Zählfläche von $9 \times 28 \mathrm{~cm}^{2}$ befanden sich gekreuzt unter der Lage II. Durch Koinzidenzen (I, II, II, III) wurden so Schauer mit annähernd vertikalem Strahlengang aussortiert.

Die Messungen wurden im Dachgeschoß des Instituts vorgenommen. Nach jeweils 12 Stunden wurde das Blei umgeschichtet. Die neu aufzulegende Bleidicke wurde durch das Los bestimmt. Die Vierfachkoinzidenzen (I, II, II, III) (Abb. 5 a) zeigen ein im Verhältnis zum Untergrund sehr hohes RossIMaximum. Außerhalb der statistischen Schwankungen liegende höhere Maxima sind nicht zu erkennen. Der Nullpunkt ist wieder durch Deckenschauer hochgedrückt. Ebenfalls registriert wurden die Zweifachkoinzidenzen (I, III), also im wesentlichen die durch das Blei gehenden Einzelstrahlen (Abb. 5 b). Die Kurve steigt zwischen 0 und $1 \mathrm{~cm}$ etwas an und zeigt dann die schnelle Absorption der weichen und die langsame der harten Komponente. Die beiden Absorptionslängen ergeben sich zu $(2,25 \pm 0,10)$ und $(205 \pm 20) \mathrm{cm} \mathrm{Pb}$. Anomalien zeigen sich auch hier nicht.

\section{Diskussion}

Mit den beschriebenen Zählrohranordnungen wurden folgende Schauertypen erfaßt: Elektronen Photonen-Kaskaden aus dem Blei, Seitenschauer und von durchdringenden Teilchen im Blei ausgelöste Schauer. Ihre relative Häufigkeit hing stark von der Geometrie und der Bleidicke ab.

Die im Blei ausgelösten Elektronenschauer wurden mitgemessen, um eine Vergleichsmöglichkeit für die bei höheren Bleidicken gesuchten Übergangseffekte zu haben. Eine quantitative Messung des Rossi-Maximums war nicht beabsichtigt. Jedoch sind die am wenigsten durch Decken- und Seitenschauer beeinträchtigten Messungen (Abb. 1 a und 5 a) be-

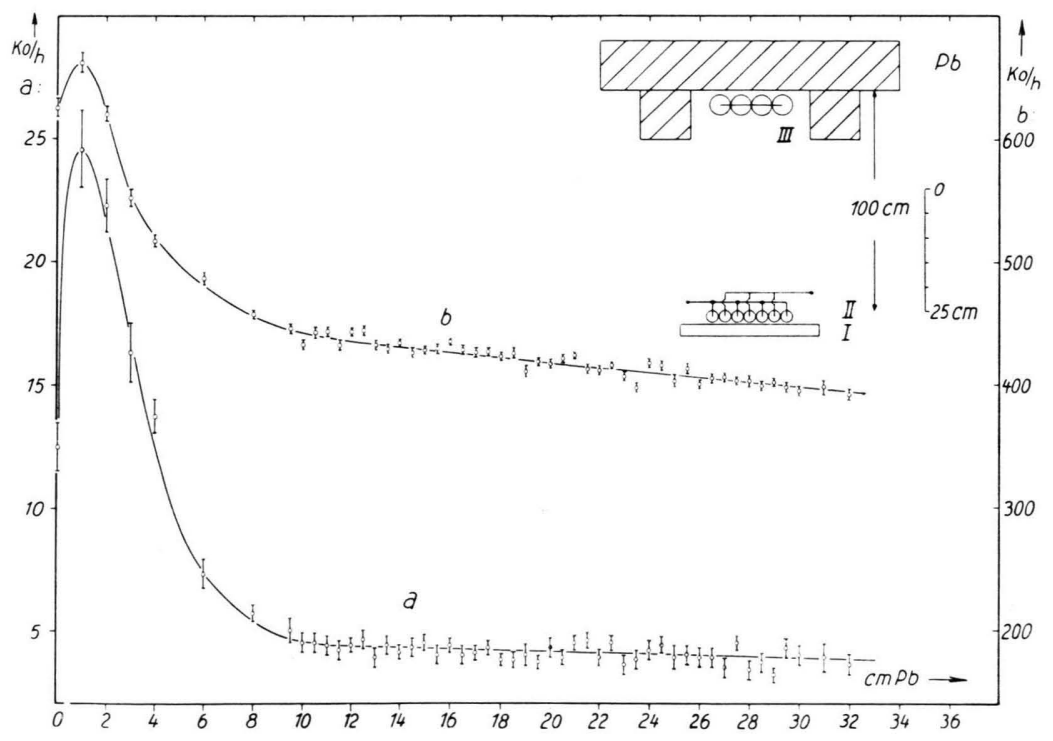

Abb. 5. Anordnung mit bevorzugt vertikalem Strahlengang. a) Schauer, die ionisierend aus dem Blei austreten: (I, II, II, III) . b) Ionisierende Einzelstrahlen: (I, III). 
züglich Höhe und Form des Rossi-Maximums mit früheren Arbeiten ${ }^{14}$ gut verträglich.

Der Abfall des Rossi-Maximums ließ sich bei allen Kurven im Bereich zwischen 2 und $10 \mathrm{~cm} \mathrm{~Pb}$ durch eine einfache Exponentialfunktion mit einer Absorptionslänge von im Mittel $(2,30 \pm 0,10) \mathrm{cm} \mathrm{Pb}$ darstellen. Gemäß Kurve $1 \mathrm{c}$ hat die Multiplizität der im Blei ausgelösten Schauer ebenfalls ein Maximum bei einigen $\mathrm{cm} \mathrm{Pb}$. Außerdem zeigt diese Kurve, $\mathrm{da} ß$ für besonders teilchenreiche Elektronenschauer das Rossi-Maximum bei größeren Bleidicken liegt als für die Gesamtheit aller Elektronenschauer. Auch das ist in qualitativer Übereinstimmung mit den theoretischen ${ }^{15}$ und experimentellen ${ }^{7}$ Ergebnissen anderer Autoren.

Seitenschauer können je nach der Zählrohranordnung mit beträchtlicher Wahrscheinlichkeit registriert werden. Bei variabler Bleischicht über einer Zusatzlage beeinflussen sie den Kurvenverlauf erheblich. Ihre Absorptionslänge ergibt sich aus den Messungen von Ziff. $3 \mathrm{zu}$ im Mittel $(2,22 \pm 0,15) \mathrm{cm} \mathrm{Pb}$. Auch bei den Seitenschauern handelt es sich also um Elektronenkaskaden.

Die Häufigkeit der bei Bleidicken über $10 \mathrm{~cm}$ ausgelösten Schauer nimmt mit wachsender Bleidicke langsam und monoton ab. Mit der Standardanordnung nach Thurn ${ }^{2}$ (Ziff. 3) werden bei $15 \mathrm{~cm} \mathrm{~Pb}$ nur ca. $65 / \mathrm{h}$ erfaßt, in Übereinstimmung mit einer früheren Abschätzung ${ }^{1}$. Ihre scheinbare Absorptionslänge ergibt sich zu im Mittel $(210 \pm 30) \mathrm{cm} \mathrm{Pb}$. Sie ist mit der Absorptionslänge durchdringender Einzelstrahlen verträglich (Literaturwert $180 \mathrm{~cm} \mathrm{~Pb}^{16}$ ). Aus den Kurven $1 \mathrm{a}$ und $2 \mathrm{~b}$ folgt unter Berücksichtigung der Nachweiswahrscheinlichkeiten für Einzelstrahlen und Schauer, daß die auslösenden Teilchen geladen sind. $\mathrm{Zu}$ dem gleichen Schluß führen die Messungen von Ziff. 3 .

Die absolute Häufigkeit der bei großen Bleidicken ausgelösten Schauer läßt sich aus den gemessenen Koinzidenzraten nur ungefähr abschätzen. Wir gehen dabei von Kurve $1 \mathrm{~b}$ aus, bei der Ionisation unter dem Blei und in einer der unteren Lagen Koinzidenzen zwischen benachbarten Zählrohren verlangt wor-

14 B. Rossi, High Energy Particles, Prentice-Hall Inc., New York 1952.

15 N. Arley, On the theory of stochastic processes and their application to the theory of cosmic radiation, Wiley, New York 1943.

16 B. Rossi, Rev. Mod. Phys. 20, 537 [1948].

17 G. PFotzer, Z. Naturforschg. 8 a, 335 [1953].

18 G. Pfotzer, Z. Naturforschg. 10 a, 718 [1955]. den waren. Nach einer Messung von Della Corte ${ }^{7}$ erfaßt man so bei $15 \mathrm{~cm} \mathrm{~Pb} 77 \%$ der Koinzidenzen zwischen beliebigen Zählrohren. Zudem sei angenommen, daß $10 \%$ aller auftreffenden Schauer nur ein Zählrohr treffen. Außerdem gehen die $n$-Strahlschauer für die Registrierung verloren, bei denen mindestens $n-1$ Schauerteilchen seitlich an den gekreuzten Lagen vorbeilaufen. Schätzt man ihren Anteil zu $(50 \pm 20) \% \mathrm{ab}$, so erhält man bei $15 \mathrm{~cm}$ $\mathrm{Pb}$ eine Schauerhäufigkeit von $\left(4_{-1}^{+2}\right) \%$ der harten Komponente. Nach Rechnungen von PFotzer ${ }^{17}$ werden in $15 \mathrm{~cm} \mathrm{~Pb}$ von $100 \mu$-Mesonen 4,5 nachweisbare Stoßelektronenschauer erzeugt. Unsere Messungen sind also mit der Annahme verträglich, daß alle bei großen Bleidicken beobachteten Schauer aus $\mu$-Mesonen-Elektronen-Schauern bestehen.

Bei den Messungen war verlangt, daß irgendwann bei der Schauerauslösung mindestens ein ionisierendes Teilchen beteiligt war. Unter dieser Voraussetzung lassen sich für die Überhöhung höherer Maxima folgende obere Schranken ableiten: $5 \mathrm{Ko} / \mathrm{h}$ für die Thurnsche Standardanordnung ${ }^{2}$ und $12 \mathrm{Ko} / \mathrm{h}$ für die Anordnung mit parallelen Zählrohren, also rd. 8 bzw. 4\% der jeweils nachgewiesenen Schauer.

Die von Pfotzer ${ }^{18}$ unabhängig gemessenen Kurven stimmen in ihrem Verlauf mit den von uns erhaltenen überein. Auslösekurven der von ionisierenden Einzelstrahlen ausgelösten Schauer hat Harding ${ }^{19,} 20$ mit einer etwas modifizierten Anordnung gekreuzter Zählrohre gemessen. Auch er fand zwischen 10 und $30 \mathrm{~cm} \mathrm{~Pb}$ einen praktisch geradlinigen Kurvenverlauf, wenn sich unter den untersten Zählrohren keine Materie befand. Andernfalls deutete sich ein breites Maximum zwischen 15 und $20 \mathrm{~cm} \mathrm{~Pb}$ an, das $\mathrm{H}_{\mathrm{AR}}$ DING auf rückwärts laufende Zerfallselektronen abgebremster $\mu$-Mesonen zurückführt. Angaben von McCusker ${ }^{21}$ wären mit Hardings Befund verträglich. Bei unseren Messungen konnte sich ein solcher Effekt nicht bemerkbar machen, da sich die untersten Zählrohre ungefähr $1 \mathrm{~m}$ oberhalb des Fußbodens befanden.

Bei Untersuchungen mit zählrohrgesteuerten Nebelkammern haben JäNecke 22,23 und McCusker und

19 J. B. Harding u. P. R. Robinson, A.E.R.E. Report N/R, 1550 [1954].

20 J. B. Harding, Proc. Phys. Soc., Lond. A 68, 354 [1955].

21 C. B. A. McCusker u. B. G. Wilson, Proc. Phys. Soc., Lond. A 68, 1086 [1955].

22 J. Jänecke, W. Bothe u. K. H. Lauterjung, Z. Naturforschg. 10 a, 794 [1955].

23 J. JäNECKE, Diss. Heidelberg (1955) u. Rundber. unveröff. Arb. Kernphys. u. Kosm. Höhenstrhlg. 
Mitarbeiter ${ }^{24}$ auch Rossı-Kurven mit Zählrohrlagen oberhalb und unterhalb des Bleischauerstrahlers aufgenommen. Auch sie erhielten eine von der Bleidicke praktisch unabhängige Zählrate, wenn sie die Zählrohre oberhalb des Bleis in Koinzidenz und die unterhalb des Bleis in Antikoinzidenz mit den unterhalb der Nebelkammer nachgewiesenen Schauern schalteten. Die Nebelkammeraufnahmen zeigen unmittelbar, daß man so nur weiche Seitenschauer erfaßt. Am hiesigen Institut untersuchte BöHning ${ }^{25}$ die Schauerauslösung in Blei mit einem Szintillationstank. Seine Ergebnisse ließen sich ebenfalls mit den bekannten Sekundärprozessen der Ultrastrahlung erklären.

Mit einer Lage paralleler Zählrohre zum Nachweis der Schauer und einer Zusatzlage dicht unter dem Blei hat Della Conte ${ }^{7}$ ebenfalls nur das RossiMaximum mit. anschließendem leichtem Abfall gemessen ${ }^{26}$. Dagegen fanden CLay und Mitarbb. ${ }^{8-11}$ sowie Allegretti und AbD EL-W $\mathrm{W}_{\text {ahab }} \mathrm{K}_{\text {halil }}{ }^{12}$ mit der gleichen allgemeinen Anordnung zum Teil ${ }^{9,10}$ scheinbar statistisch gesicherte höhere Maxima. Ein statistisch gesichert scheinendes 2. Maximum erhielten auch Kameda und Miura ${ }^{27}$ durch Ausblendung ionisierender Primärer bei Koinzidenzraten von der Größenordnung 0,1 bis höchstens $1 \mathrm{~h}^{-1}$. Eine ältere

24 P. D. McCormack, C. B. A. McCusker u. B. G. Wilson, Proc. Phys. Soc., Lond. A 68, 1088 [1955].

25 M. Böhning, Diplomarbeit Heidelberg (1956).

26 Della Corte erhält zwar durch Differenzenbildung aus den Meßkurven (7, Fig. 3) nach Anbringen einiger Korrekturen ein 2. Maximum für die Schauer mit genau 2 Teilchen (7, Fig. 5), doch liegt dieses ersichtlich innerhalb der Fehler.
Messung von Bernardini ${ }^{28}$ läßt dagegen, ebenfalls bei Forderung ionisierender Primärteilchen, keine Andeutung eines 2. Maximum erkennen.

Die früheren Ergebnisse von ThURN ${ }^{2-4}$ und KRÄMER $^{5}$ lassen sich mit den von uns gewonnenen nicht vereinen. Wir glauben, daß die vorliegenden und die in ${ }^{1}$ beschriebenen genauer und zuverlässiger sind. Auch Jánossy ${ }^{29}$ hat kürzlich mit einer Nachbildung der Thursschen Standardanordnung (ohne Zusatzlagen) die Messungen von Thurn und KräMer nicht bestätigen können. $\mathrm{NooH}^{13}$ findet mit der Anordnung von Abb. 5 zwischen 0 und $48 \mathrm{~cm} \mathrm{~Pb}$ eine Serie von 10 Maxima. Dafür wissen wir keine Erklärung.

Fenyves und Haimand ${ }^{30}$ gaben eine Absorptionskurve der Ultrastrahlung an, die Maxima bei ungefähr 18 und $27 \mathrm{~cm} \mathrm{~Pb}$ aufweist und oberhalb $30 \mathrm{~cm} \mathrm{~Pb}$ steil abfällt. Heyland und Duncanson ${ }^{31}$ fanden dagegen in Übereinstimmung mit der von uns gemessenen Kurve (Abb. $5 \mathrm{~b}$ ) oberhalb $10 \mathrm{~cm}$ $\mathrm{Pb}$ einen langsamen und monotonen Abfall.

Herrn Prof. Dr. W. Bothe danken wir für die Anregung zu dieser Arbeit und für viele Ratschläge und Diskussionen. Der Deutschen Forschungs g e meinschaft sei für die Bereitstellung von Apparaten gedankt.

27 T. Kameda u. I. Miura, Progr. Theor. Phys. 5, 323 [1950].

28 G. Bernardini, Ric. sci. (1) 5, 533 [1934].

29 L. Jánossy u. L. NAGY, Z. Naturforschg. 11 a, 312 [1956]. Wir danken Herrn Prof. Jánossy für die freundliche Úbersendung des Manuskripts.

30 E. Fenyves u. O. Haimann, Nature, Lond. 165, 244 [1950].

31 G. R. Heyland u. W. E. Duncanson, Nature Lond. 167, 895 [1951] u. Proc. Phys. Soc., Lond. A 66, 33 [1953]. 